\title{
RNA-Seq profile of flavescence dorée phytoplasma in grapevine
}

\author{
Simona Abbà ${ }^{*}$, Luciana Galetto ${ }^{1}$, Patricia Carle ${ }^{2,3}$, Sébastien Carrère ${ }^{4,5}$, Massimo Delledonne ${ }^{6}$, Xavier Foissac ${ }^{2,3}$, \\ Sabrina Palmano ${ }^{1}$, Flavio Veratti ${ }^{1}$ and Cristina Marzachì ${ }^{1}$
}

\begin{abstract}
Background: The phytoplasma-borne disease flavescence dorée is still a threat to European viticulture, despite mandatory control measures and prophylaxis against the leafhopper vector. Given the economic importance of grapevine, it is essential to find alternative strategies to contain the spread, in order to possibly reduce the current use of harmful insecticides. Further studies of the pathogen, the vector and the mechanisms of phytoplasma-host interactions could improve our understanding of the disease. In this work, RNA-Seq technology followed by three de novo assembly strategies was used to provide the first comprehensive transcriptomics landscape of flavescence dorée phytoplasma (FD) infecting field-grown Vitis vinifera leaves.

Results: With an average of 8300 FD-mapped reads per library, we assembled 347 sequences, corresponding to 215 annotated genes, and identified 10 previously unannotated genes, 15 polycistronic transcripts and three genes supposedly localized in the gaps of the FD92 draft genome. Furthermore, we improved the annotation of 44 genes with the addition of $5^{\prime} / 3^{\prime}$ untranslated regions. Functional classification revealed that the most expressed genes were either related to translation and protein biosynthesis or hypothetical proteins with unknown function. Some of these hypothetical proteins were predicted to be secreted, so they could be bacterial effectors with a potential role in modulating the interaction with the host plant. Interestingly, qRT-PCR validation of the RNA-Seq expression values confirmed that a group II intron represented the FD genomic region with the highest expression during grapevine infection. This mobile element may contribute to the genomic plasticity that is necessary for the phytoplasma to increase its fitness and endorse host-adaptive strategies.

Conclusions: The RNA-Seq technology was successfully applied for the first time to analyse the FD global transcriptome profile during grapevine infection. Our results provided new insights into the transcriptional organization and gene structure of FD. This may represent the starting point for the application of high-throughput sequencing technologies to study differential expression in FD and in other phytoplasmas with an unprecedented resolution.
\end{abstract}

Keywords: Flavescence dorée phytoplasma, RNA-Seq, Vitis vinifera, Group II intron, Hypothetical proteins, qRT-PCR

\section{Background}

Grapevine flavescence dorée phytoplasma (FD) is one of the most severely damaging diseases affecting European vineyards. This quarantine pest continues to have a significant economic impact not only in the two major European wine-producing countries, Italy and France, but also in Spain, Switzerland, Portugal, Austria, Croatia, Slovenia, Serbia and Hungary [1]. The cicadellid leafhopper Scaphoideus titanus Ball is the only known

\footnotetext{
* Correspondence: s.abba@ivv.cnr.it

${ }^{1}$ Istituto per la Protezione Sostenibile delle Piante, IPSP-CNR, Strada delle Cacce 73, I-10135 Torino, Italy

Full list of author information is available at the end of the article
}

vector that transmits the disease from grapevine to grapevine [2]. Symptoms appear in early summer and increase in incidence and severity until harvest. These symptoms consist of drying of berry peduncles and consecutive shrivelling or drying of berries, leaf discolouration and downward leaf curling [3]. Consequently, grapevine vitality and yields are reduced, and the production of wine is irreparably compromised.

Phylogenetical analyses indicated that FD can be divided into two taxonomic $16 \mathrm{~S}$ groups, $16 \mathrm{SrV}-\mathrm{C}$ and $16 \mathrm{SrV}-\mathrm{D}[4,5]$, and three genetic clusters according to the sequence of the map gene: map-FD1 (including isolate FD70), map-FD2 (including isolates FD92 and FD-D) and 
map-FD3 (including isolate FD-C) [6]. The three clusters show different geographical distributions. In Piedmont, which is one of the most renowned Italian wine-making regions, both FD-C and FD-D isolates have been detected [7]. In this region, the local administration spent nearly $€$ 1.5 million per year from 1999 to 2003 in disease control programs. Additionally, in 2005 the Italian government and the European Union spent $€ 34$ million to refund growers for yield losses and replanting [8]. Better knowledge of the molecular interaction between the pathogen and its hosts is thus essential to develop new and sustainable control strategies to reduce the non-target impacts of the compulsory insecticide treatments against the vector.

New high-throughput "omics" technologies, such as whole transcriptome sequencing (RNA-Seq) and highresolution mass spectrometry, allow simultaneous examination of thousands of genes, transcripts, proteins, and metabolites, opening new possibilities towards marker discovery and genome-wide identification of signalling molecules, protein functions and interactions. Such technologies were recently applied also to the study of plant-phytoplasma interactions, but in most of these cases only the plant responses to the phytoplasma infection have been investigated, leaving the phytoplasma perspective almost unexplored [9-16]. Only two reports took advantage of the new high-throughput technologies to describe the transcriptional and proteome landscape of phytoplasmas: Ji and colleagues provided the first valuable dataset of mulberry dwarf phytoplasma proteins by a shotgun proteomics approach [17] and, more recently, Siewert and colleagues [18] combined RNA-Seq and shotgun proteomics to provide insights into the expressed genes of 'Candidatus Phytoplasma mali' infecting graft-inoculated Nicotiana occidentalis leaves. Microarrays were instead used for the first global gene expression study on phytoplasmas, which evaluated the changes in gene expression during 'Candidatus Phytoplasma asteris' "host-switching" between plant to insect hosts [19].

In the present study, RNA-Seq provided the first comprehensive transcriptomics landscape of FD phytoplasma infecting field-grown cv. Barbera grapevines. An annotated draft genome of the FD92 isolate, covering $85 \%$ of the 671 kbp chromosome, has been recently produced using a combination of 454 pyrosequencing and Illumina/Solexa $[20,21]$. In the absence of a complete FD genome sequence, different approaches to separate FD-mapping reads from the eukaryotic ones followed by assemblies and recursive merging of sequence datasets were used to reconstruct the FD transcripts. In this way, previously unannotated regions, polycistronic transcripts, 5'/3' UTR regions and non-coding RNAs (ncRNAs) have been identified. Moreover, genes potentially involved in plant-phytoplasma interactions were selected to validate the RNA-Seq results by qRT-PCR on field-grown grapevines infected by either $16 \mathrm{SrV}-\mathrm{C}$ or $-\mathrm{D}$ isolates.

\section{Results}

\section{Phytoplasma detection, strain characterization and quantification}

Diagnostic assays confirmed the presence of FD and the absence of stolbur 'Bois noir' (BN) phytoplasma in all three grapevine leaf samples used in this study (120, B68 and B75). In addition, qRT-PCR assays on the most common viruses reported in Piedmont vineyards revealed the presence of Grapevine rupestris stem pitting-associated virus in all samples and Grapevine fleck virus in sample 120 and B75.

On the basis of the TaqI-RFLP profiles of the ribosomal $16 \mathrm{~S}$ gene, samples 120 and B68 were identified as $16 \mathrm{SrV}-\mathrm{C}$ isolates and sample B75 as a $16 \mathrm{SrV}-\mathrm{D}$ isolate (data not shown). In the three samples, the phytoplasma titer expressed as number of FD cells/500 $\mathrm{mg}$ of plant tissue ranged from $5.81 \mathrm{E}+05$ to $6.32 \mathrm{E}+06$.

\section{Relative quantification of grapevine and phytoplasma mRNAs}

The ratio between the grapevine and the phytoplasma transcripts was evaluated by qRT-PCR, considering the expression of two single-copy genes: FD secY and grapevine ubiquitin. The transcriptional levels of these two genes were estimated to be 6 and 32000 copy number, respectively, so FD transcripts represented nearly $0.02 \%$ of the total grapevine messengers.

\section{Data coverage}

Total RNA extracted from sample 120 was split in two parts, named sample 120 and sample 120E.

The two samples were analysed separately as technical replicates in independent sequencing runs, generating two sets of total FD transcriptome profiles.

A total of $125,813,174$ and $129,412,231$ paired-end (PE) reads $(2 \times 100 b p)$ with an average insert size of $153 \mathrm{bp} \pm 39$ bp was generated from libraries 120 and $120 \mathrm{E}$, respectively.

The quality control and trimming resulted in two filtered sets (95\% of reads passed the control) assembled with three different strategies. The effectiveness of each assembly approach was evaluated and compared to the others in order to choose the best performer and obtain the best description of the FD transcriptome (Additional file 1).

\section{Reads assembly}

As a first assembly approach, reads from libraries 120 and $120 \mathrm{E}$ were first mapped to the FD92 draft genome (Table 1) and then assembled. The number of PE reads mapping in the antisense orientation to the FD92 predicted coding sequences (Table 1, third row) was below 
Table 1 Number of PE reads from libraries $120 \mathrm{E}$ and 120 that mapped at least once to the coding and non-coding regions of the FD92 genome

\begin{tabular}{lccc}
\hline & 120E & 120 & Total (120E + 120) \\
\hline PE Reads mapping concordantly to the FD92 rRNA/tRNA molecules & 1864 & 4026 & 5890 \\
PE reads mapping concordantly to the FD92 annotated CDSs in the sense orientation & 2891 & 3068 & 5959 \\
PE reads mapping concordantly to the FD92 annotated CDSs in the antisense orientation & 99 & 62 & 161 \\
PE reads mapping concordantly to the FD92 IGRs & 2431 & 2323 & 4754 \\
PE reads mapping concordantly to the FD92 genome & 7285 & 9479 & 16764 \\
\hline
\end{tabular}

the theoretical maximum number of spurious cDNAs generated during the second-strand synthesis of the reverse transcription (calculated to be 145 reads for library $120 \mathrm{E}$ and 189 for library 120), so they were not further analyzed.

In each library nearly $0.01 \%$ of the total reads were mapped, confirming the ratio obtained by qRT-PCR. The two separate assemblies obtained from the libraries (dataset 1 and dataset 2) were compared to evaluate the technical reproducibility. Some sequences were unique to each dataset, i.e. they did not find any significant hits in others, whereas many other sequences found at least one significant hit with $100 \%$ identity across the alignment, but with a different length. These results suggested the importance of dealing with at least two technical replicates when studying the genome-wide transcriptome profile of an organism whose sequences represent a very small fraction of the total RNA-Seq library. A merged dataset (dataset 3) was obtained from the assembly of these two initial datasets.

The second assembly approach consisted in merging reads from the two libraries, mapping them to the FD92 genome and then assembling them altogether. The resulting dataset (dataset 4) was compared to dataset 3 to determine the best assembly strategy. Sequences from dataset 4 included all the sequences of dataset 3 and were generally longer than the corresponding sequences of dataset 3. Therefore, the second assembly approach proved to be far more efficient than the first one. A new merged dataset (dataset 5) was obtained from the assembly of dataset 3 and 4 .

As a final approach, reads from the two merged libraries were first mapped to the $V$. vinifera genome. The Vitisunmapped reads, which represented nearly $0.7 \%$ of each library, were then de novo assembled. The resulting sequences with significant similarities to known Mollicutes genes (dataset 6) were compared to dataset 5 . No unique sequences were identified in dataset 6 , but still there were differences in length between some matching sequences from the two datasets. The third assembly approach was found to be less efficient than the second one, both in terms of number and in terms of the average length of the assembled sequences. However, it was adopted in combination with the second approach to obtain a more accurate description of the FD transcriptome.

A final comprehensive FD transcriptome dataset of 347 sequences with an average length of 294 bp was created by assembling dataset 5 and 6 and used for further analyses.

\section{Comparison of RNA-Seq transcripts to FD92 annotated genes}

334 out of 347 RNA-Seq sequences corresponded to 215 FD92 annotated genes (18 were full-length protein-coding transcripts) (Additional file 2). Of the remaining 13 sequences, 10 were present in the FD92 genome but the matching regions were not yet annotated, and three did not find any match to the FD92 genome but showed significant similarities to Poinsettia branch-inducing phytoplasma sequences (Additional file 2). PCR analyses with specific primers demonstrated that these three sequences truly belonged to the phytoplasma genome (Additional file 3), so they were probably localized in the gaps of the FD92 draft genome.

The 10 unannotated transcripts (Additional file 2) were further analyzed to determine whether they showed significant similarities to known Mollicutes protein-coding sequences or previously characterized ncRNAs. After querying the NCBI "nr" and the Rfam databases, four of them showed significant similarities to three phytoplasma hypothetical proteins and a translation initiation factor IF-3. In addition, contig12 and contig6 showed significant similarities (expected value, E-value $\leq 1 \mathrm{E}-5$ ) to ncRNAs, namely the catalytically active RNA of a group II intron and the RNA component of a bacterial ribonuclease (RNase) $\mathrm{P}$ class $\mathrm{B}$, respectively. The remaining four unannotated transcripts showed putative ORFs spanning the whole length or a part of the nucleotide sequence, but the corresponding predicted proteins did not have any significant similarity to other bacterial sequences.

RNA-Seq data also provided the opportunity to extend the length of some transcripts compared to the automatic computer annotation of FD92 genes: 34 sequences were extended by at least 1 nucleotide upstream of the predicted translation start (adding a potential 5' untranslated region - 5' UTR) and 11 sequences were extended downstream of the predicted stop codon in 11 sequences 
(adding a potential 3' UTR) (Additional file 2). The gene coding for the $30 \mathrm{~S}$ ribosomal subunit protein $\mathrm{S} 8 \mathrm{rps} \mathrm{H}$ was extended in both directions.

Transcripts that i) mapped to IGRs between two non-overlapping consecutive CDS on the same strand and ii) partially overlapped the two genes on the same strand were considered as parts of polycistronic transcripts (Additional file 2, see FD92 annotated transcripts with two hits). According to our data, there were at least 15 polycistronic transcripts in FD transcriptome, most of them involving ribosomal proteins. Excluding operons that were made up only of ribosomal transcripts, most polycistronic transcripts contained two or three genes (Table 2).

As sample 120 showed a $16 \mathrm{SrV}-\mathrm{C}$ restriction profile whereas FD92 is a $16 \mathrm{SrV}-\mathrm{D}$ isolate [6], polymorphisms identified at the nucleotide level in the comparison between RNA-Seq data and the FD92 genome could be exploited for isolate genotyping (Additional file 4).

\section{Phytoplasma in silico gene expression}

The expression levels of a dataset that included the FD92 annotated genes extended by the newly identified 5' UTRs and 3' UTRs and the newly identified transcripts, both coding and non-coding, are shown in Additional file 5. The most expressed transcript, named contig12, corresponded to the catalytic RNA component of a group II intron, which was present in the FD92 genome in at least two slightly divergent copies. It is important to underline that one of these two copies was artificially truncated due to its position at the end of a genomic contig. Even if divided by two, the transcriptional level of this catalytic intron remained the highest in both RNA-Seq libraries. Interestingly, a blastx analysis of contig12 revealed the presence, at the 3' end of the sequence, of the first 27 amino acids of a putative transposase tra 5 for insertion sequence element IS150 (GenBank:WP_015637618.1). A further analysis of the complete copy corresponding to contig12 revealed that it did not contain any internal protein-encoding open reading frames, so it supposedly belonged to the category of the group II "ORF-less" introns. No sequences with significant similarity to ltrA, an intron II reverse transcriptase already annotated in other phytoplasmas genomes, were found either in the RNA-Seq data or in other regions of the FD92 genome. Additionally, the tra5 fragment identified in contig 12 was truncated not only in the transcript but also in the corresponding genome region.

The first 20 most expressed transcripts included also the immunodominant protein Imp, the variable membrane protein VmpA, the ribozyme component of a bacterial Rnase P class B, six hypothetical proteins, the GroEL chaperonin and nine proteins involved in the translation process (Table 3).

Expressed transcripts were classified by comparing sequences against the manually curated KEGG GENES database (Additional file 5). Excluding the generic functional category named "Metabolic pathways", the most represented categories during Vitis infection were those related to translation and protein metabolism (ribosome, tRNA and amino acids biosynthesis), DNA metabolism (pyrimidine and purine metabolism, DNA replication, DNA repair) and carbon metabolism (including glycolysis/gluconeogenesis) (Figure 1). In particular, all the enzymes of the glycolysis pathway were transcribed. Transport, protein export and secretion categories were also well represented with a total of 16 transcripts. Unfortunately, nearly $30 \%$ of the total assembled transcripts were not functionally classified.

Where possible, protein-coding transcripts were associated to GO terms by Blast2GO (Additional file 5). In order to identify whether any GO term was over-/ under-represented in the list of the 20 most expressed transcripts, an Enrichment Analysis with two-tailed Fisher's exact test $(\mathrm{P}<0.05)$ was performed against all the expressed genes. The over-represented $\mathrm{GO}$ terms were related to translation and protein metabolism ("structural constituent of ribosome", "ribosome biogenesis", "translation", "unfolded protein binding", "ribosome"), whereas the only under-represented GO term was "catalytic activity" (Figure 2 and Additional file 5).

Table 2 Polycistronic transcripts as identified by the RNA-seq assembly

\begin{tabular}{ccc}
\hline $\begin{array}{c}\text { Number of genes } \\
\text { in operon }\end{array}$ & Gene 1 & Gene 2 \\
\hline 2 & fba-flado_0482_0037 & Cof-flado_0482_0038 \\
2 & pdhB-flado_8220_0009 & Ctg8220_0011015_0011245_f2-flado_8220_0010 \\
3 & grpE-flado_0031_0008 & dnaK-flado_0031_0007 \\
2 & Ctg8084_0025861_0026808_r1-flado_8084_0018 & mnmE-flado_8084_0017 \\
3 & rpsG-flado_6343_0020 & fusA-flado_6343_0021 \\
2 & tdk-flado_6333_0005 & tadA-flado_6333_0004 \\
2 & trmD-flado_0314_2012 & rplS-flado_0314_0009 \\
2 & hup-flado_0234_0030 & rpmG-flado_0234_0031
\end{tabular}


Table 3 The top 20 highly expressed FD genes during V. vinifera infection

\begin{tabular}{|c|c|c|}
\hline locus ID & Description & RPK (mean) \\
\hline Contig12 & Group II catalytic intron & 2749.2 \\
\hline Ctg0426_0019664_0020134_f2_flado_0426_0012 & Imp & 889.1 \\
\hline Ctg0234_0028748_0029098_f2_flado_0234_0025 & Hypothetical protein with cold-shock binding domain & 548.8 \\
\hline Ctg0482_0000001_0000355_r3_flado_0482_0001 & Hypothetical protein with $1 \mathrm{TMD}+\mathrm{SP}$ & 460.8 \\
\hline rplP-flado_0067_0029 & 50 S ribosomal subunit protein L16 & 95.6 \\
\hline tuf-flado_6343_0022 & Elongation factor EF-Tu & 95.6 \\
\hline Contig6 & Bacterial Rnase P class B & 80.3 \\
\hline Ctg7221_0018383_0018643_r1_flado_7221_0014 & Hypothetical protein with $1 \mathrm{TMD}+\mathrm{SP}$ & 54.7 \\
\hline Ctg5304_0020454_0020660_f3_flado_5304_0019 & Hypothetical protein with 1 TMD & 48.6 \\
\hline vmpA-flado_0482_0026 & Variable membrane protein A & 48.3 \\
\hline Ctg7221_0002401_0002754_f1_flado_7221_0002 & Hypothetical protein with $1 \mathrm{TMD}+\mathrm{SP}$ & 47.9 \\
\hline rpsU-flado_5304_0006 & $30 S$ ribosomal subunit protein $\$ 21$ & 46.0 \\
\hline rplV-flado_0067_0031 & 50 S ribosomal subunit protein L22 & 43.9 \\
\hline rpsK-flado_0067_0011 & 30 ribosomal subunit protein S11 & 41.8 \\
\hline Ctg0314_0004067_0004231_f2_flado_0314_0005 & Hypothetical protein & 41.7 \\
\hline groL-flado_0426_0003 & Chaperonin GroEL & 40.3 \\
\hline rp|N-flado_0067_0026 & 50 S ribosomal subunit protein L14 & 37.1 \\
\hline rpsM-flado_0067_0012 & 30 S ribosomal subunit protein S13 & 37.1 \\
\hline fusA-flado_6343_0021 & Elongation factor EF-G & 34.5 \\
\hline rplB-flado_0067_0033 & $50 S$ ribosomal subunit protein $\mathrm{L} 2$ & 33.4 \\
\hline
\end{tabular}

Transcription levels are expressed as the mean of RPK (reads per kilobase of transcript) values obtained in the two expression libraries. The first column (locus ID) reported the IDs of the annotated FD92 genes, with the only exception of contig12, which was obtained in this study. A brief gene description and the mean of reads per kilobase of transcript (RPK) are also reported. TMD = transmembrane domain predicted by TMHMM; SP = signal peptide predicted by SignalP-HMM v. 3 .

\section{Validation of RNA-Seq expression values by qRT-PCR}

Target genes for qRT-PCR assays were selected taking into account three criteria: 1) a wide range of in silico expression levels, from the highest (contig12 with $\mathrm{RPK}=2749)$ to the lowest (rpoD with RPK =0), 2) the possible involvement in the host-phytoplasma interactions and 3) the annotation as "hypothetical proteins" of unknown function. Fifteen target genes were selected: contig12 as the most expressed transcript, two known surface-exposed proteins (Imp and VmpB) [22-25], five hypothetical proteins (comp83, comp115, comp100, comp126, PAM266), a protein known to regulate the excretion of virulence factors in other bacteria (SpoVG) [26], a protein involved in the secretion pathway (ftsY), two proteolytic enzymes potentially contributing to virulence (ysdC and tldD), a protein involved in the defence mechanisms against oxidative stress (osm), the RNA polymerase sigma factor $\mathrm{rpoD}$ and an $\mathrm{ABC}$ transporter (CoABC) (Table 4).

The existence of transcripts corresponding to contig12 and to the hypothetical proteins was first confirmed by RT-PCR on the same RNA used for the RNA-seq library construction (data not shown). Expression of the selected FD genes was examined in three FD-infected grapevine samples: sample 120, B68 and B75. To estimate the expression level of the selected FD transcripts, the mRNA absolute quantity was compared to the phytoplasma titer measured in the corresponding sample. The primers list, the corresponding amplification conditions and efficiencies, melting peak temperatures and correlation coefficients of qRT-PCR reactions were reported in Additional file 6.

For each gene, the average Expression Index (EI, $\mathrm{N}^{\circ}$ of transcript in single FD cells) in the three grapevine samples was calculated (Table 4 and Additional file 5). The reliability and reproducibility of the expression values of the selected transcripts were confirmed by the squared correlation coefficient $(\mathrm{r} 2=0.98)$ calculated between the qRT-PCR and the RPK values (Figure 3 ).

According to the Welch-one way test, the mean of expression levels was significantly different among target transcripts $(P<0.05)$. Contig12 was the most abundant transcript in all three grapevine samples, irrespective of their restriction profiles, with an average EI significantly higher than all the other genes.

\section{Discussion}

Application of deep sequencing technologies to specifically study the transcriptome of intracellular obligate endosymbiont bacteria has been attempted in very few 


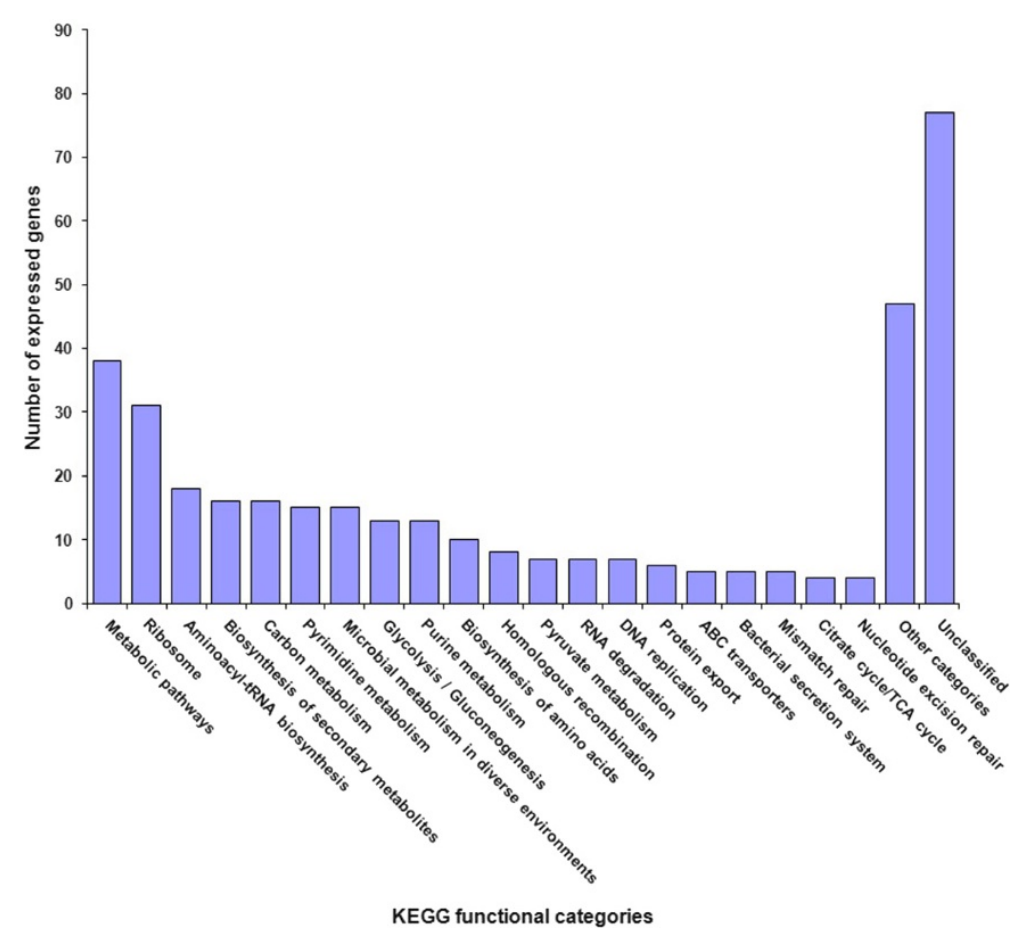

Figure 1 Distribution of assembled transcripts into functional categories according to KEGG classification. Number of expressed genes (y-axis) associated to KEGG pathways (x-axis).

cases, because the selection of prokaryotic transcripts in a multitude of eukaryotic RNAs makes the accurate representation of the bacterial transcriptome particularly challenging. To our knowledge, the only overall gene expression studies conducted so far on phytoplasmas was that of Siewert and colleagues [18] on 'Ca. P. mali'. Other whole-transcriptome analyses of obligate intracellular bacteria were, for instance, concerned with A. phagocytophilum [27], Lawsonia intracellularis [28] and Wolbachia in symbiosis with Onchocerca volvulus [29]. In almost all of these studies, including the one on 'Ca. P. mali', hosts were artificially infected and kept in laboratory conditions. The study on Wolbachia was so far the only one in which an obligate intracellular bacterium was analyzed under natural conditions, i.e. in nematodes living in cattle raised in field. In all cases, a bacterium-enrichment procedure was undertaken by selecting particular cell types/tissues and/or by adopting specific experimental procedures (selective hybridization, host rRNA depletion or polyA transcript depletion). In our work, samples were collected in field from a Piedmontese vineyard in late July and the pathogen-enrichment process consisted of coupling the RNA extraction from leaf midribs to eukaryotic rRNA depletion.

In 'Ca. P. mali', the RNA-Seq approach resulted in 468 mapped reads, corresponding to 132 expressed genes [18]. In our study, deep sequencing of a FD-infected grapevine sample followed by three assembly approaches allowed us to obtain an average of 8300 FD-mapped reads per library, which represented a better coverage of the reference transcriptome. As in many other genomicscale transcriptomics surveys, also in this study RNA-Seq

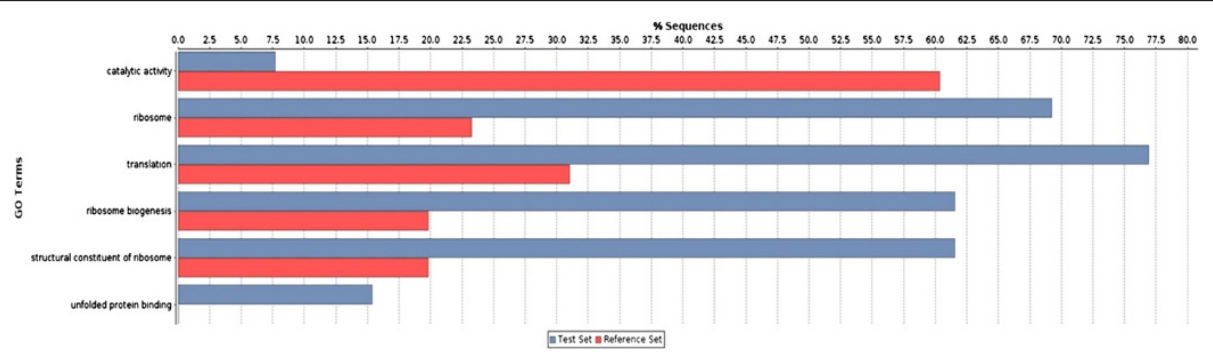

Figure 2 Enrichment analysis of GO terms calculated by Fisher's exact test. Reference set (red) is represented by all the FD expressed genes, whereas the test set (blue) is represented by the top 20 highly expressed FD genes. 
Table 4 Transcripts selected for validation by qRT-PCR

\begin{tabular}{|c|c|c|c|c|}
\hline locus ID & Abbreviation & Description & RPK (mean) & El (mean) \\
\hline Contig12 & contig12 & Group II intron & 2749.2 & 10.9 \\
\hline Ctg0426_0019664_0020134_f2_flado_0426_0012 & Imp & Imp & 889.1 & 3.5 \\
\hline Ctg0234_0028748_0029098_f2_flado_0234_0025 & comp83 & Hypothetical protein with cold-shock binding domain & 548.8 & 2.1 \\
\hline Ctg7221_0018383_0018643_r1_flado_7221_0014 & comp100 & Hypothetical protein & 54.7 & 1.1 \\
\hline Ctg0314_0004067_0004231_f2_flado_0314_0005 & comp115 & Hypothetical protein with $1 \mathrm{TMD}+\mathrm{SP}$ & 41.7 & 1.4 \\
\hline Ctg0482_0033346_0033975_f1_flado_0482_0023 & spoVG & septation protein & 32 & 0.5 \\
\hline ysdC-flado_4539_0017 & ysdC & $\beta$-glucanase & 29.2 & 0.5 \\
\hline Ctg0338_0006451_0007086_f1_flado_0338_0005 & comp126 & Hypothetical protein with $1 \mathrm{TMD}+\mathrm{SP}$ & 23.3 & 0 \\
\hline vmpB-flado_0031_0011 & $\mathrm{vmpB}$ & Variable membrane protein $B$ & 13.1 & 0.1 \\
\hline tldD-flado_0426_0020 & $\mathrm{tldD}$ & Metalloprotease & 12.2 & 0.2 \\
\hline ftsY-flado_0482_0016 & fstY & Signal recognition particle receptor & 9.1 & 0.1 \\
\hline Ctg0314_0007223_0007996_f2_flado_0314_0008 & PAM266 & Hypothetical protein (similar to PAM266) & 8 & 0.1 \\
\hline CbiO2-flado_0067_0008 & COABC & Cobalt transporter ATP-binding subunit & 5 & 0.2 \\
\hline Ctg5304_0021069_0021488_f3_flado_5304_0021 & osm & OsmC-like protein & 4.8 & 0.1 \\
\hline rpoD-flado_4539_0007 & rpoD & RNA polymerase sigma factor & 0 & 0.5 \\
\hline
\end{tabular}

Selected genes are listed according to the IDs of the FD92 annotated genes (locus ID), with the only exception of contig12, which was obtained in this study. The abbreviation used in this work, a brief gene description, the mean of reads per kilobase of transcript (RPK) and the mean of the expression indices (El, $\mathrm{N}^{\circ}$ of transcript per FD cell) are reported.

proved to be an extremely powerful technique for a) the detection and quantification of previously unannotated transcripts, b) the discovery of the polycistronic organization of some transcripts and c) the addition of $5^{\prime} / 3^{\prime}$ UTRs to protein-coding genes. This kind of data becomes extremely precious especially when dealing with obligate intracellular bacteria, which can neither be grown under laboratory conditions nor genetically manipulated.

In our work, we assembled 347 sequences corresponding to 219 protein-coding genes (4 were previously unannotated) and we found that 273 FD92 genes showed at least one mapped read in both libraries. Mastronunzio and colleagues identified 82 A. phagocytophilum expressed genes [27], whereas Vannucci and colleagues reported that 754 protein-coding genes of $L$. intracellularis showed at least one mapped read [28]. Our results are close to the number of expressed transcripts reported for other obligate intracellular bacteria, despite the fact that the number of reads mapping to those genomes was higher, ranging from hundreds of thousands to millions of reads.

Analyzing global transcriptional levels, genes related to ribosomal structure and biogenesis, transcription and protein biosynthesis were the most expressed in FD, as

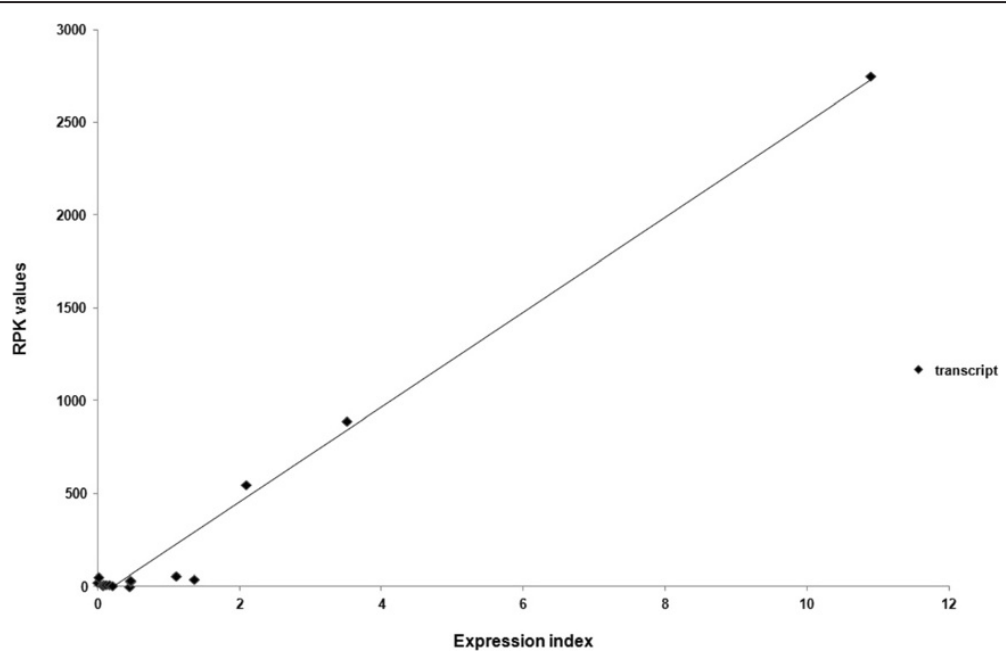

Figure 3 Correlation between RNA-Seq and qRT-PCR expression data. Plot of the transcriptional levels of the 15 selected genes expressed as RPK values (y-axis) and Expression Index values ( $x$-axis). $R^{2}=0.98(P<0.05)$. 
well as in L. intracellularis [28]. This result, along with the absence of highly expressed genes related to the DNA replication machinery or cell cycle, suggests that, in late July, when the FD titer has been demonstrated to be the highest [30], phytoplasma cells had already undergone extensive cycles of replication and growth. Therefore, most of the energy could be rerouted to protein synthesis. Interestingly, the chaperone GroEL was included in the list of the FD most expressed genes. Its remarkable ability to rescue non-productive protein conformations is particularly crucial in obligate endosymbiotic bacteria, in which the fixation of slightly deleterious mutations as a result of their distinctive lifestyle often affects the functional conformation of proteins [31].

Excluding the transcription/translation-related proteins, almost all the other proteins in the list of FD top 20 highly expressed genes are known or predicted membrane proteins. Imp and Vmp proteins are well known cellsurface proteins and several studies suggested they might have a prominent role in the host-phytoplasma interaction [22-25]. In addition, two hypothetical proteins (Ctg0482_0000001_0000355_r3_flado_0482_0001 and Ctg7221_0018383_0018643_r1_flado_7221_0014) were predicted to have one transmembrane region and a cleavage site, so they are likely to remain attached to the FD membrane after secretion. The nucleotide polymorphisms we observed in some membrane protein-coding genes between the FD92 genome and our RNA-Seq data are consistent with the necessary adaptations of the bacterium to its complex and changing relationship with the host. These differences could be exploited for studying the FD genetic diversity within different strains.

Besides genes related to protein synthesis, the functional classification highlighted a high percentage of unclassified genes. This was probably due to a) the lack of a good functional annotation for some known phytoplasma proteins, such as SpoVG, Imp, VmpA, $\mathrm{VmpB}$, etc...., and b) the presence of many hypothetical proteins whose functions are still unknown and thus not yet included in databases.

It is noteworthy that the abundance of hypothetical proteins (six in total) among the FD most expressed genes is in accordance to the results obtained in L. intracellularis [28] and Wolbachia [29], in which seven and four out of the 20 most expressed genes encoded hypothetical proteins, respectively. Even in 'Ca. P. mali' the highest number of RNA-Seq reads was assigned to a conserved hypothetical integral membrane protein [18]. Therefore, such hypothetical proteins of unknown function should be the main targets for future analyses to elucidate their potential role in host-bacterial interactions.

Other known potential phytoplasma effectors, such as SAP-like proteins [32-34], HflB proteases and AAA+
ATPases [35], superoxide dismutases [36], proteins of the Sec-dependent secretion pathway [37], were expressed, but not listed among the most expressed genes in FD.

The most striking results were the high expression levels of two ribozymes that were not previously annotated in the FD92 genome: the RNA component of a bacterial RNase P class B and the catalytic RNA of a group II intron. Bacterial RNases P are made up of two components: a catalytic RNA and a polypeptide chain. Together they function as an endoribonuclease that removes the precursor sequence from the $5^{\prime}$ end of a primary tRNA transcript to generate mature tRNAs [38]. The high level of transcription of the RNA component of this ribonucleoprotein complex was consistent with the high expression of genes related to translation, as tRNA maturation is an essential process for protein biosynthesis.

Widespread in prokaryotes and in organelles of fungi, plants and lower eukaryotes, group II introns are genetic retroelement capable of self-splicing and inserting into DNA sites [39]. They typically consist of a ribozyme, which catalyzes splicing events, and a protein, which takes part both in splicing and insertion events. In prokaryotes, the protein component, which shows endonuclease, reverse transcriptase and maturase domains, is encoded by the intron itself and is essential for both retrohoming and retrotransposition [39]. In the FD92 genome two almost identical copies of this group II intron were identified so far. One of these was artificially truncated at the $3^{\prime}$ due to its position at the end of a genomic contig, so only the other copy was analyzed in more detail. The complete genomic copy of this group II intron was very similar (77\% identity, E-value $=4 \mathrm{E}-39$ ) to the Onion yellows phytoplasma intron named OYPI1 (GenBank:AP006628 Region: 388234-390749) [40], but, unlike OYPI1, it seemed to be an ORF-less intron, as neither a reverse transcriptase nor any other protein-coding gene was detected within the intron sequence. As already hypothesized for Onion yellows phytoplasma ORF-less OYPI2 (GenBank: AP006628 Region: 544682-545416) [41], isolate FD92 could also harbor in the gaps of its unfinished genome at least one full-length group II intron that may act in trans on the other ORF-less group II intron(s). It has, in fact, been shown that group II introns that became fragmented by genome rearrangements in eukaryotic organelles have the capacity to ligate independently transcribed coding sequences, splice accurately in vivo and finally produce a functional mRNA [42]. The presence of a tra5 remnant at the 3 ' of contig12 suggested that the absence of the retrotranscriptase in this copy could indeed be associated with some genome rearrangements. Finally, we cannot exclude that the missing part of the other copy of group II intron could encode itself a functional reverse transcriptase. 
The high transcriptional levels of contig12 and the presence in the FD92 genome of other proteins usually associated with group II intron mobility, such as a complete recA and a DEAD-box protein [43,44], may indicate that the retroelement can be fully functional.

To our knowledge, this is the first report of the expression of a phytoplasma group II intron during plant infection. The high transcriptional levels of this gene were confirmed by qRT-PCR on the same sample used for the RNA-Seq library construction (sample 120) and on other two grapevine samples (samples B68 and B75), even though they belonged to two different $16 \mathrm{~S}$ restriction profiles. Hypothesizing that contig12 could really be part of a functional retroelement, it may contribute to the genomic plasticity that is necessary for the phytoplasma to increase its fitness and, ultimately, adapt to its host. Mobile elements, in general, are known to play key roles during the emergence of host-adaptive strategies in bacteria [45] and group II introns, in particular, have been demonstrated to be responsible of some recent genomic rearrangements in the bacterial endosymbiont Wolbachia [46].

Fourteen additional genes, including highly expressed hypothetical proteins and genes with possible involvements in the host-bacterium interactions, were examined by qRT-PCR. The positive correlation on a linear regression model between qRT-PCR and RPK values and the high R-squared value demonstrated that RNA-Seq data properly estimated the expression levels of the selected genes.

\section{Conclusions}

The RNA-seq technology was successfully applied for the first time to analyse the FD global transcriptome profile during grapevine infection. Our results provided new insights into the FD gene structure, transcriptional organization and expression levels with an unprecedented resolution for phytoplasmas. The low number of FD-mapped reads has not prevented us from improving the genome annotation or from providing a reliable view of the FD transcriptome, but it was a limitation when exploring the antisense transcription. The strand-specific RNA-seq could have shed some light on the FD antisense transcriptional activity, which is totally unexplored. However, because the number of reads mapping to FD annotated CDSs in the antisense orientation was lower than the possible error threshold of the technique, any consideration on this subject would have been merely speculative. A further enrichment for phytoplasma sequences would be necessary not only for this purpose, but also when comparing variable conditions, e.g. the phytoplasma transcriptome in response to the host plant and to the insect vector. A higher number of phytoplasma-mapped reads could, in fact, increase the probability of finding statistically significant differences in the expression profiles. As shown in this work, the use of at least two technical replicates per sample is also essential to provide a broader and more reliable picture of the transcriptional landscape, especially when the genome coverage is low.

\section{Methods}

\section{Plant material}

Leaf samples from symptomatic field-grown cv. 'Barbera' grapevines (growth stage: 6-7 leaves separated) were collected in Cocconato (Piedmont, Italy) in late July 2013. The vineyard, which consisted of approximately 8600 plants arranged in 76 north-south running rows, has been monitored since 2007 for phytoplasma infection, and so a detailed map of the sanitary status of the plants (healthy, infected and recovered) was available at the beginning of this study. Plants were regularly treated with fungicides and no typical symptoms of fungal diseases were observed during sampling. Molecular assays were used to detect the presence of FD and BN phytoplasmas [47]. Leaves infected only by FD were retained for further studies, whilst samples with mixed infections were excluded from this work. Among the FD-positive plants, sample 120 from row 62 was chosen for the RNA-Seq analysis.

\section{Nucleic acid extraction}

For each samples, total RNA and DNA were extracted from $500 \mathrm{mg}$ of pooled plant material. Total RNA was extracted following the protocol described by Chang and colleagues [48] and treated with RNase-free DNase I (Applied Biosystems, Foster City, CA, USA) to avoid residual DNA contamination. After nucleic acid precipitation with $\mathrm{LiCl}$, the RNA-depleted supernatant was retained for total DNA extraction.

\section{Phytoplasma detection, strain characterization and quantification}

For FD and BN diagnosis, 40 ng of DNA were used in direct PCR with universal primers P1/P7 [49]. Reaction products were used as templates in nested PCRs driven by primers R16 (V and I) F1/R1 [50].

For FD strain characterization, P1/P7 amplicons were also used as templates in nested PCRs with primers M1/B6 [4]. Nested PCR amplicons were then digested with TaqI endonuclease (Thermo Fisher Scientific, Walthem, MA, USA) for $1 \mathrm{~h}$ at $37^{\circ} \mathrm{C}$ and the restriction profiles visualized after electrophoresis on ethidium bromide-stained acrylamide gels.

\section{Preparation of strand-specific RNA-Seq libraries}

Libraries construction and sequencing on the Illumina HiSeq 1000 were performed at the Centro di Genomica Funzionale, Dipartimento di Biotecnologie, Università degli Studi di Verona, Verona, Italy. The Epicentre Ribo-Zero ${ }^{\mathrm{Tm}}$ 
Magnetic Kit (Plant Leaf) was used to remove rRNA from 3.5 micrograms of total RNA. The rRNA-depleted total RNA fractions were used to generate two directional libraries with the Illumina TruSeq Stranded Total RNA preparation kit. According to the kit manual, more than $98 \%$ of the mapped reads should return accurate strand origin information, whereas the remaining reads may represent non-specific background.

\section{Sequence analysis}

Settings used for each of the following computer programs were specified in Additional file 7.

Before read mapping and assembly, poor quality data were filtered out by Trimmomatic v. 0.30 [51].

The partial FD92 draft genome (cluster FD2) [52] used in this work is available upon request at https://iant.toulouse. inra.fr/F.doree.

Bowtie v. 0.12.7 [53] was used to map reads to the FD92 genome as well as to the assembled transcripts for the evaluation of gene expression levels.

Tophat v. 1.4.1 [54] was preferred to Bowtie for mapping reads to the $12 \mathrm{X}$ Vitis vinifera genome [55], because it can identify splice junctions between exons.

Given the low amount of FD-mapped PE reads (thousands) compared to other projects dealing with transcriptomes of free-living bacteria (millions), we decided to retain, only for the transcriptome assembly, the single-end (SE) reads, i.e. those PE reads in which only one read of the couple passed the quality check. SE reads were not considered for the quantitative analysis. The minimum assembled contig length to be reported by Trinity assembler (Trinityrnaseq_r20131110) [56] was set to $150 \mathrm{bp}$ to avoid losing short protein-coding RNAs, considering that 49-51 amino acid-long proteins are quite common in phytoplasmas (e.g. GenBank:WP_011161061.1, GenBank:NP_950971.1, GenBank:AAO61980, GenBank: AAO61987).

After each Trinity assembly, the assembled sequences were first analysed by blastn [57] against the FD92 genome. Assembled nucleotide sequences were considered as significantly similar to the FD92 genome if they showed at least 95\% identity across the alignment with an E-value $\leq 1 \mathrm{E}-20$. Those without a match were then analysed by blastn against the NCBI "nt database" to discard sequences belonging to plant plastidial/mitochondrial genomes or other bacterial genomes. Finally, sequences with no significant similarities to the NCBI "nt database" were analysed by blastx [57] against the NCBI "nr database" to retain only those with significant similarities $(E$-value $\leq 0.0001)$ to Mollicutes predicted proteins. This last step was used to find coding sequences that could potentially be localized in the gaps of the unfinished FD92 genome.

Sequence datasets obtained with different assembly strategies were merged using cap3 (version:4/15/05) [58]. After each cap3 assembly, sequences were again checked against the FD92 genome to avoid misassemblies.

A dataset enriched in FD-mapping PE reads was submitted to Sequence Read Archive (SRA) (http://www. ebi.ac.uk/ena/data/view/PRJEB6982).

\section{In silico phytoplasma gene expression}

The expression level of each transcript was calculated by an ad-hoc Bash wrapper script calling Bowtie v. 0.12.7 and expressed as number of reads per kilobase of transcript (RPK). Given the low amount of FD-mapped reads, we decided to use RPK instead of RPKM (number of reads per kilobase per million reads mapped). RPK values were calculated using only $\mathrm{PE}$ reads that mapped concordantly, unambiguously and on the correct strand. To compare the RPK values of the two libraries, the RPK values obtained from library $120 \mathrm{E}$ were multiplied by a factor of 1.3, which took into account the different number of FD-mapped reads in the two libraries.

The expression level of each gene that was predicted to be part of a polycistronic transcript was evaluated separately. Only genes with mapped reads in both libraries were considered expressed in order to provide more accurate values of their transcriptional rates. The RPK values were thus provided as the mean of the RPK values of library 120 and library 120E.

$23 \mathrm{~S}$ and $16 \mathrm{~S}$ ribosomal RNAs were excluded from the list of expressed genes, since they are usually the most abundant RNAs in all species.

\section{Prediction of signal peptides and transmembrane domains}

SignalP v. 3.0 [59] with HMM method was used to predict the presence of signal peptides (minimum probability threshold $=0.9$ ).

Transmembrane helices were predicted by TMHMM Server v. 2.0 [60].

\section{Functional analyses of expressed genes}

KAAS server [61] based on bi-directional best hit information was used to identify the functional properties and the biological roles of expressed genes.

The Gene Ontology (GO) functional classification and the following enrichment analysis with Fisher's Exact Test (test set: the top 20 highly expressed FD genes during $V$. vinifera infection; reference set: all the expressed FD genes during $V$. vinifera infection ) were performed by the Blast2GO suite [62].

\section{qPCR and qRT-PCR assays: FD titer, gene expression and MRNA relative quantification}

All qPCR and qRT-PCR reactions were carried out in a CFX Connect ${ }^{\mathrm{TM}}$ Real-Time PCR Detection System 
(Bio-Rad) supported by the CFX Manager ${ }^{\mathrm{Tw}}$ Software, version 3.0.

FD titer and gene expression analyses were performed on the same sample used for RNA-Seq (sample 120) and on two additional FD-infected samples, named B75 and B68, collected in the proximity of sample 120 , from rows 75 and 68, respectively. These two samples were chosen according to their $16 \mathrm{~S}$ restriction profiles: $\mathrm{B} 68$ showed the same $16 \mathrm{~S}-\mathrm{C}$ profile as sample 120 , whilst $\mathrm{B} 75$ belonged to the FD-D cluster. To minimize potential variability due to environmental conditions, the three plant samples were collected on the same day.

Plants in Cocconato vineyard were regularly treated with fungicides and no typical symptoms of fungal diseases were observed during sampling. The three samples used in this work were tested by qRT-PCR for the presence of eight viruses, which are all common in Piedmont vineyards: Grapevine Virus A (GVA), Grapevine Virus B (GVB), Grapevine Fanleaf Virus (GFLV), GFkV, Grapevine Leaf-Roll-Associated Viruses (GLRaV) 1, 2 and 3 and Arabis mosaic virus (ArMV) $[47,63]$.

FD titer was calculated as already described by Roggia and colleagues [30] and expressed as number of FD cells/500 mg of leaf sample.

For qRT-PCR assays, cDNA was synthesized from $500 \mathrm{ng}$ of total RNA using the High Capacity cDNA Reverse Transcription Kit (Applied Biosystems, Foster City, CA, USA).

External standard curves were produced using serial dilutions of plasmids carrying fragments of the 15 selected genes amplified by conventional PCR from total DNA of FD-C-infected periwinkle (Piedmont isolate). In particular, primers by Galetto and colleagues were used for the amplification of the Imp gene [64]. Plasmids carrying amplicons were used to set up standard curves, ranging from $10^{8}$ to $10^{4}$ transcript copy number. The number of plasmid copies per microlitre was derived from the concentration measured at the Nanodrop spectrophotometer, according to the equation described by Osborn and Smith [65].

SYBR Green-based qRT-PCR protocols were optimized and the final mix contained $25 \mathrm{ng}$ of $\mathrm{CDNA}, 1 \mathrm{x} \mathrm{iQ}^{\mathrm{m}}$ SYBR $^{\circ}$ Green Supermix (Bio-Rad, Life Science Research, Hercules, CA, USA), $300 \mathrm{nM}$ primers, sterile double distilled water to a final volume of $10 \mu \mathrm{l}$. Reaction conditions were as follows: $5 \mathrm{~min}$ at $95^{\circ} \mathrm{C}$ and 45 cycles of $30 \mathrm{sec}$ at $95^{\circ} \mathrm{C}$ followed by $1 \mathrm{~min}$ at the optimized annealing and extension temperatures (Additional file 6). Samples were run in duplicate together with four tenfold serial dilutions (from $10^{2}$ to $10^{8}$ transcript copy number) of the corresponding standard plasmid. The complete qRT-PCR mix with total RNA and sterile distilled water instead of cDNA were used as negative controls on each plate. Melting curves were produced at the end of the PCR to assess the reaction specificity: the PCR products were heated to $95^{\circ} \mathrm{C}$ for $1 \mathrm{~min}$, cooled at $65^{\circ} \mathrm{C}$ and held at that temperature for $1 \mathrm{~min}$ and then slowly heated back to $95^{\circ} \mathrm{C}$ at a rate of $0.5^{\circ} \mathrm{C} /$ cycle.

An Expression Index (EI) of each target phytoplasma gene was calculated for each sample as the ratio between the FD mRNA absolute quantity and the phytoplasma titer measured in the corresponding sample. The Shapiro-Wilk test and the Bartlett test revealed that the EIs of each gene in different samples were normally distributed, but did not show equal variances. The Welch-one way ANOVA for normally distributed and heteroscedastic data, followed by the Waller-Duncan test, was then used for multiple comparisons. All statistical analysis were performed with the R Stats package v 3.1.1 (http://www.R-project.org).

The same secY primers and amplification conditions used for calculating the FD titer were also used to estimate the relative abundance of phytoplasma mRNA in sample 120 . In this case, the single-copy ubiquitin gene of grapevine (GenBank:FQ378362.1) was used instead of the 18S rDNA gene. Ubiquitin primers by Gutha and colleagues [66] were used to determine the transcript levels of this gene.

\section{Additional files}

\begin{abstract}
Additional file 1: Comparison among the three assembly strategies
Table reports: how the two libraries were used for the assembly (separated or in combination), the name of the resulting dataset, the datasets that were compared two-by-two at the end of each assembly approach, the number of assembled sequences for each dataset, the average length of sequences belonging to each dataset, the number of unique sequences for each dataset and the name of the dataset obtained after the merging with cap3. * and $\S$ indicate that FD-mapped reads and unmapped Vitis reads were used, respectively.
\end{abstract}

Additional file 2: Comparing RNA-Seq assembled sequences to the FD92 genome. Tables report: RNA-Seq assembled sequences that matched to FD92 annotated genes (sheet: "FD92 annotated genes"); assembled transcripts with no match to the FD92 genome, but with significant similarities to other phytoplasmas proteins (sheet: "hits to other phytoplasmas"); RNA-Seq assembled sequences that matched to the FD92 genome, but were not annotated (sheet: "FD92 unannotated genes"). \# and § indicate assembled transcripts that added either the 5' UTR or the 3' UTR region to previously annotated FD92 genes, respectively; * indicates genes that are part of a polycistronic transcript.

Additional file 3: PCR products of the three RNA-Seq sequences with no match to the FD92 genome. Figure shows the amplicons obtained from healthy $(\mathrm{H})$ and FD-C infected $(\mathrm{I})$ periwinkle maintained in laboratory conditions. $M=1$ kb plus marker (Thermo Fisher Scientific, Walthem, MA, USA).The primers used for amplifications were: contig $1 \mathrm{f}$ 5'- GCCTGATAGAAAAAAAGTAG -3' and contig1r 5' - TTAGGAGAAATTTCTCCTGTAT - 3' (Annealing temperature $=59^{\circ} \mathrm{C}$ ); contig2f 5'- GAGAATCTGTAATGTATAAGG -3'and contig2r 5' - TCAATATCTTCAG GAGTAGG - 3'(Annealing temperature $=60^{\circ} \mathrm{C}$ ); novitis_comp95067f 5'- TGTGGCGATAACAAGAGCAA - 3' and novitis_comp95067r 5'- TGTGCA TAACCTTATCTCCTGC $-3^{\prime}$ (Annealing temperature $=62^{\circ} \mathrm{C}$ ).

Additional file 4: Assembled transcripts that showed less than $100 \%$ identity at the nucleotide level with the FD92 annotated genes. Maltose/maltodextrin-binding periplasmic protein malE is present twice in the list because two assembled transcripts corresponded to two different parts of the corresponding FD92 gene. Most differences corresponded to non-synonymous substitutions, so these polymorphisms 
could potentially affect the protein conformation and/or functionality. ${ }^{*}$ known/predicted membrane proteins; ${ }^{\S} \mathrm{nCRNA}$; TMD $=$ transmembrane domain predicted by TMHMM.

Additional file 5: Transcriptional levels and functional classification of expressed genes. Tables report: the locus ID (IDs of the FD92 annotated genes and the transcripts assembled in this work), the gene names, the RPK values of all the expressed genes in libraries 120 and $120 \mathrm{E}$ separately, the mean of the two RPK values, the GO classification, the enzymes codes (sheet: "expressed genes"); the locus ID, the abbreviations used in this work, the RPK values in libraries 120 and 12 OE, the mean of RPK values, the El values in 120, B68 and B75 samples, and the mean of the El values for the 15 selected genes (sheet: "selected genes RPK \& El"); the KEGG functional classification of the expressed gens, as performed by KAAS (sheet: "KEGG functional classification"); the GO Enrichment Analysis with Fisher's exact test $(P<0.05)$ of the top 20 highly expressed genes, as performed by Blast2GO (sheet: "GO enrichment analysis").

Additional file 6: PCR/qPCR/qRT-PCR primers and amplification conditions. Table reports primers sequences, amplification conditions and reaction parameters (for qRT-PCRs only) of all the PCR analyses carried out in this work.

Additional file 7: Parameters used for bioinformatics analysis. File reports for each software the list of parameters that were set to values different from the default ones.

\section{Competing interests}

The authors declare that they have no competing interests.

\section{Authors' contributions}

$S A, L G, S P$ and $C M$ conceived the study and participated in its design and coordination. SA extracted RNA and performed the RNA-Seq data analysis, LG performed all the qPCR and qRT-PCR analyses. PC, SC and XF set up the iANT annotation platform for the FD92 draft genome. FV carried out the experiments to determine the strain assignment of the grapevine samples. SA and LG wrote the manuscript. SP, CM, SC, XF and MD critically revised the manuscript, and all authors have read and given approval of the version to be published.

\section{Acknowledgments}

This work was part of the "Nebbiolo Genomics" project sponsored by Fondazione Cassa di Risparmio di Cuneo, Italy. The FD92 genome draft was obtained with the support of INRA and the Interprofessional Council for Bordeaux's Wine (CIVB). The authors also acknowledge the financial support of the Cost Actions FA0807 ("Integrated Management of Phytoplasma Epidemics in Different Crop Systems") and FA0806 ("Plant virus control employing RNA-based vaccines: A novel non-transgenic strategy"). We thank Marta Vallino and Piero Caciagli (IPSP-CNR, Torino, Italy) for discussions; Mario Altieri (University of Verona, Verona, Italy) and Stefano Ghignone (IPSP-CNR, Torino, Italy) for technical assistance in bioinformatics analyses; Rafael Montero (Universitat de les Illes Balears, Palma, Spain) for providing the primers and the plasmid to quantify the grapevine ubiquitin transcription levels. Special thanks to Giancarlo Birello (IRCrES-CNR, Torino, Italy) for building up and managing the bioinformatics server and to Dylan Beal (MontpellierSupAgro, Montpellier, France) for English revision of the text.

\section{Author details}

${ }^{1}$ Istituto per la Protezione Sostenibile delle Piante, IPSP-CNR, Strada delle Cacce 73, I-10135 Torino, Italy. ${ }^{2}$ INRA, UMR1332 Biologie du Fruit et Pathologie, 71 avenue Edouard Bourlaux, CS20032, F-33882 Villenave d'Ornon, Cedex, France. ${ }^{3}$ Université de Bordeaux, UMR1332 Biologie du Fruit et Pathologie, 71 avenue Edouard Bourlaux, CS20032, F-33882 Villenave d'Ornon, Cedex, France. ${ }^{4}$ INRA, Laboratoire des Interactions

Plantes-Microorganismes (LIPM), UMR441, Castanet-Tolosan F-31326, France. ${ }^{5}$ CNRS, Laboratoire des Interactions Plantes-Microorganismes (LIPM), UMR2594, Castanet-Tolosan F-31326, France. ${ }^{6}$ Dipartimento di Biotecnologie, Università degli Studi di Verona, Strada le Grazie 15, I-37134 Verona, Italy.

Received: 2 September 2014 Accepted: 4 December 2014 Published: 11 December 2014

\section{References}

1. EFSA Panel on Plant Health: Scientific opinion on pest categorisation of Grapevine Flavescence dorée. EFSA J 2014, 12:3851.

2. Weintraub PG, Beanland L: Insect vectors of phytoplasmas. Annu Rev Entomol 2005, 51:91-111.

3. Margaria P, Rosa C, Marzachì C, Turina M, Palmano S: Detection of flavescence dorée phytoplasma in grapevine by reverse-transcription PCR. Plant Dis 2007, 91:1496-1501.

4. Martini M, Murari M, Mori N, Bertaccini A: Identification and epidemic distribution of two flavescence dorée-related phytoplasmas in Veneto (Italy). Plant Dis 1999, 83:925-930.

5. Davis RE, Dally EL: Revised subgroup classification of group $16 \mathrm{SrV}$ phytoplasmas and placement of flavescence dorée associated phytoplasmas in two distinct subgroups. Plant Dis 2001, 85:790-797.

6. Arnaud G, Malembic-Maher S, Salar P, Bonnet P, Maixner M, Marcone C, Boudon-Padieu E, Foissac X: Multilocus sequence typing confirms the close genetic interrelatedness of three distinct flavescence dorée phytoplasma strain clusters and group $16 \mathrm{SrV}$ phytoplasmas infecting grapevine and alder in Europe. Appl Environ Microbiol 2007, 73:4001-4010.

7. Martini M, Botti S, Marcone C, Marzachì C, Casati P, Bianco PA, Benedetti R, Bertaccini A: Genetic variability among flavescence dorée phytoplasmas from different origins in Italy and France. Mol Cell Probes 2002, 16:197-208.

8. Belli G, Bianco PA, Conti M: Grapevine yellows in Italy: past, present and future. J Plant Pathol 2010, 92:303-326.

9. Albertazzi G, Milc J, Caffagni A, Francia E, Roncaglia E, Ferrari F, Tagliafico E, Stefani E, Pecchioni N: Gene expression in grapevine cultivars in response to Bois Noir phytoplasma infection. Plant Sci 2009, 176:792-804.

10. Hren M, Nikolic P, Rotter A, Blejec A, Terrier N, Ravnikar M, Dermastia M, Gruden K: 'Bois noir' phytoplasma induces significant reprogramming of the leaf transcriptome in the field grown grapevine. BMC Genomics 2009, 10:460.

11. Ehya F, Monavarfeshani A, Mohseni Fard E, Karimi Farsad L, Khayam Nekouei M, Mardi M, Salekdeh GH: Phytoplasma-responsive microRNAs modulate hormonal, nutritional, and stress signalling pathways in Mexican lime trees. PLoS One 2013, 8:e66372.

12. Mou H-Q, Lu J, Zhu S-F, Lin C-L, Tian G-Z, Xu X, Zhao W-J: Transcriptomic analysis of Paulownia infected by Paulownia witches'-broom Phytoplasma. PLoS One 2013, 10:e77217.

13. Liu LY, Tseng HI, Lin CP, Lin YY, Huang YH, Huang CK, Chang TH, Lin SS: High-throughput transcriptome analysis of the leafy flower transition of Catharanthus roseus induced by peanut Witches'-broom phytoplasma infection. Plant Cell Physiol 2014, 55:942-957.

14. Luge T, Kube M, Freiwald A, Meierhofer D, Seemüller E, Sauer S: Transcriptomics assisted proteomic analysis of Nicotiana occidentalis infected by 'Candidatus Phytoplasma mali' strain AT. Proteomics 2014, 14:1882-1889.

15. Monavarfeshani A, Mirzaei M, Sarhadi E, Amirkhani A, Khayam Nekouei M, Haynes PA, Mardi M, Salekdeh GH: Shotgun proteomic analysis of the Mexican lime tree infected with 'CandidatusPhytoplasma aurantifolia'. $J$ Proteome Res 2012, 12:785-795.

16. Gai YP, Li YQ, Guo FY, Yuan CZ, Mo YY, Zhang HL, Wang H, Ji XL: Analysis of phytoplasma-responsive sRNAs provide insight into the pathogenic mechanisms of mulberry yellow dwarf disease. Sci Rep 2014, 4:5378.

17. Ji X, Gai Y, Lu B, Zheng C, Mu Z: Shotgun proteomic analysis of mulberry dwarf phytoplasma. Proteome Sci 2010, 8:20.

18. Siewert C, Luge T, Duduk B, Seemüller E, Büttner C, Sauer S, Kube M: Analysis of expressed genes of the bacterium 'Candidatus phytoplasma Mali' highlights key features of virulence and metabolism. PLoS One 2014, 9:e94391.

19. Oshima K, Ishii Y, Kakizawa S, Sugawara K, Neriya Y, Himeno M, Minato N, Miura C, Shiraishi T, Yamaji Y, Namba S: Dramatic transcriptional changes in an intracellular parasite enable host switching between plant and insect. PLoS One 2011, 6:e23242.

20. Carle P, Malembic-Maher S, Arricau-Bouvery N, Desqué D, Eveillard S, Carrère S, Foissac X: Flavescence dorée phytoplasma genome: a metabolism oriented towards glycolysis and protein degradation. Bull Insectol (Supplement) 2011, 64:S13-S14.

21. Malembic-Maher S, Constable F, Cimerman A, Arnaud G, Carle P, Foissac X, Boudon-Padieu E: A chromosome map of the Flavescence dorée phytoplasma. Microbiology 2008, 154:1454-1463.

22. Kakizawa S, Oshima K, Jung HY, Suzuki S, Nishigawa H, Arashida R, Miyata S, Ugaki M, Kishino H, Namba S: Positive selection acting on a surface membrane protein of the plant-pathogenic phytoplasmas. J Bacteriol 2006, 188:3424-3428. 
23. Boonrod K, Munteanu B, Jarausch B, Jarausch W, Krczal G: An immunodominant membrane protein (Imp) of 'Candidatus phytoplasma mali' binds to plant actin. MPMI 2012, 25:889-895

24. Cimerman A, Pacifico D, Salar P, Marzachì C, Foissac X: Striking diversity of vmp1, a variable gene encoding a putative membrane protein of the stolbur phytoplasma. Appl Environ Microbiol 2009, 75:2951-2957.

25. Murolo S, Marcone C, Prota V, Garau R, Foissac X, Romanazzi G: Genetic variability of the stolbur phytoplasma vmp1 gene in grapevines, bindweeds and vegetables. J Appl Microbiol 2010, 109:2049-2059.

26. Schulthess B, Bloes DA, Francois $P$, Girard M, Schrenzel J, Bischoff M, Berger-Bachi B: sigmaB-dependent yabJ-spoVG operon involved in the regulation of extracellular nuclease, lipase and protease expression in Staphylococcus aureus. J Bacteriol 2011, 193:4954-4962.

27. Mastronunzio JE, Kurscheid S, Fikrig E: Post-genomic analyses reveal development of infectious Anaplasma phagocytophilum during transmission from ticks to mice. J Bacteriol 2012, 194:2238-2247.

28. Vannucci F, Foster D, Gebhart C: Laser microdissection coupled with RNA-sec analysis of porcine enterocytes infected with an obligate intracellular pathogen (Lawsonia intracellularis). BMC Genomics 2013, 14:421

29. Darby AC, Armstrong SD, Bah GS, Kaur G, Hughes MA, Kay SM, Koldkjær P, Rainbow L, Radford AD, Blaxter ML, Tanya VN, Trees AJ, Cordaux R, Wastling JM Makepeace BL: Analysis of gene expression from the Wolbachia genome of a filarial nematode supports both metabolic and defensive roles within the symbiosis. Genome Res 2012, 22:2467-2477.

30. Roggia C, Caciagli P, Galetto L, Pacifico D, Veratti F, Bosco D, Marzachì C: Flavescence dorée phytoplasma titer in field-infected Barbera and Nebbiolo grapevines. Plant Pathol 2014, 63:31-41.

31. Moran NA: Accelerated evolution and Muller's rachet in endosymbiotic bacteria. Proc Natl Acad Sci U S A 1996, 93:2873-2878.

32. Bai X, Correa VR, Toruño TY, Ammar ED, Kamoun S, Hogenhout SA: AY-WB phytoplasma secretes a protein that targets plant cell nuclei. MPMI 2008, 22:18-30.

33. Sugio A, Kingdom HN, MacLean AM, Grieve VM, Hogenhout SA: Phytoplasma protein effector SAP11 enhances insect vector reproduction by manipulating plant development and defense hormone biosynthesis. Proc Natl Acad Sci U S A 2011, 108:E1254-E1263.

34. MacLean AM, Sugio A, Makarova OV, Findlay KC, Grieve VM, Tóth R, Nicolaisen M, Hogenhout SA: Phytoplasma effector SAP54 induces indeterminate leaf-like flower development in Arabidopsis plants. Plant Physiol 2011, 157:831-841.

35. Seemüller E, Sule S, Kube M, Jelkmann W, Schneider B: The AAA+ ATPases and $\mathrm{HflB} / \mathrm{FtsH}$ proteases of 'Candidatus phytoplasma mali': phylogenetic diversity, membrane topology, and relationship to strain virulence. MPMI 2013, 26:367-376.

36. Miura C, Sugawara K, Neriya Y, Minato N, Keima T, Himeno M, Maejima K, Komatsu K, Yamaji Y, Oshima K, Namba S: Functional characterization and gene expression profiling of superoxide dismutase from plant pathogenic phytoplasma. Gene 2012, 510:107-112.

37. Bai X, Zhang J, Ewing A, Miller SA, Jancso Radek A, Shevchenko DV, Tsukerman K, Walunas T, Lapidus A, Campbell JW, Hogenhout SA: Living with genome instability: the adaptation of phytoplasmas to diverse environments of their insect and plant hosts. J Bacterio/ 2006, 188:3682-3696.

38. Evans D, Marquez SM, Pace NR: RNase P: interface of the RNA and protein worlds. Trends Biochem Sci 2006, 31:333-341

39. Tourasse NJ, Stabell FB, Reiter L, Kolstø AB: Unusual group II introns in bacteria of the bacillus cereus group. J Bacterio/ 2005, 187:5437-5451.

40. Oshima K, Kakizawa S, Nishigawa H, Jung HY, Wei W, Suzuki S, Arashida R, Nakata D, Miyata S, Ugaki M, Namba S: Reductive evolution suggested from the complete genome sequence of a plant-pathogenic phytoplasma. Nat Genet 2004, 36:27-29.

41. Simon DM, Clarke NAC, McNeil BA, Johnson I, Pantuso D, Dai L, Chai D, Zimmerly S: Group II introns in Eubacteria and Archaea: ORF-less introns and new varieties. RNA 2008, 14:1704-1713.

42. Bonen L: Trans-splicing of pre-mRNA in plants, animals, and protists. FASEB J 1993, 7:40-46.

43. Cousineau B, Lawrence S, Smith D, Belfort M: Retrotransposition of a bacterial group II intron. Nature 2000, 404:1018-1021.

44. Del Campo M, Tijerina P, Bhaskaran H, Mohr S, Yang Q, Jankowsky E, Russell R, Lambowitz AM: Do DEAD-Box proteins promote group II intron splicing without unwinding RNA? Mol Cell 2007, 28:159-166.
45. Toft C, Andersson SGE: Evolutionary microbial genomics: insights into bacterial host adaptation. Nat Rev Genet 2010, 11:465-475.

46. Leclercq S, Giraud I, Cordaux R: Remarkable abundance and evolution of mobile group II introns in wolbachia bacterial endosymbionts. Mol Biol Evol 2011, 28:685-697.

47. Margaria $P$, Turina M, Palmano S: Detection of Flavescence dorée and Bois noir phytoplasmas, Grapevine leafroll associated virus- 1 and -3 and Grapevine virus $A$ from the same crude extract by reverse transcription-RealTime Taqman assays. Plant Pathol 2009, 58:838-845.

48. Chang S, Puryear J, Cairney J: A simple and efficient method for isolating RNA from pine trees. Plant Mol Biol Rep 1993, 11:113-116.

49. Schneider B, Seemüller E, Smart CD, Kirkpatrick BC: Phylogenetic Classification of Plant Pathogenic Mycoplasma-Like Organisms or Phytoplasmas. In Molecular and Diagnostic Procedures in Mycoplasmology. Edited by Razin S, Tully JG. San Diego, CA: Academic Press; 1995:369-380.

50. Lee IM, Gundersen DE, Hammond RW, Davis RE: Use of mycoplasmalike organism (MLO) group-specific oligonucleotide primers for nested-PCR assays to detect mixed-MLO infections in a single host plant. Phytopathology 1994, 84:559-566.

51. Bolger AM, Lohse M, Usadel B: Trimmomatic: a flexible trimmer for Illumina sequence data. Bioinformatics 2014, 30:2114-2120.

52. Carle P, Malembic-Maher S, Arricau-Bouvery N, Desqué D, Eveillard S, Carrère S, Foissac X: 'Flavescence dorée' phytoplasma genome: a metabolism oriented towards glycolysis and protein degradation. Bull Insectol 2011, 64:S13-S14.

53. Langmead B, Trapnell C, Pop M, Salzberg S: Ultrafast and memory-efficient alignment of short DNA sequences to the human genome. Genome Biol 2009, 10:R25.

54. Trapnell C, Pachter L, Salzberg SL: TopHat: discovering splice junctions with RNA-Seq. Bioinformatics 2009, 25:1105-1111.

55. Grimplet J, Van Hemert J, Carbonell-Bejerano P, Diaz-Riquelme J, Dickerson J, Fennell A, Pezzotti M, Martinez-Zapater J: Comparative analysis of grapevine whole-genome gene predictions, functional annotation, categorization and integration of the predicted gene sequences. BMC Res Notes 2012, 5:213.

56. Grabherr MG, Haas BJ, Yassour M, Levin JZ, Thompson DA, Amit I, Adiconis X, Fan L, Raychowdhury R, Zeng Q, Chen Z, Mauceli E, Hacohen N, Gnirke A, Rhind N, di Palma F, Birren BW, Nusbaum C, Lindblad-Toh K, Friedman N, Regev A: Full-length transcriptome assembly from RNA-Seq data without a reference genome. Nat Biotech 2011, 29:644-652.

57. Altschul SF, Gish W, Miller W, Myers EW, Lipman DJ: Basic local alignment search tool. J Mol Biol 1990, 215:403-410.

58. Huang $X$, Madan A: CAP3: a DNA sequence assembly program. Genome Res 1999, 9:868-877.

59. Dyrløv Bendtsen J, Nielsen H, von Heijne G, Brunak S: Improved prediction of signal peptides: SignalP 3.0. J Mol Biol 2004, 340:783-795.

60. Krogh A, Larsson BR, von Heijne G, Sonnhammer ELL: Predicting transmembrane protein topology with a hidden markov model: application to complete genomes. J Mol Biol 2001, 305:567-580.

61. Moriya Y, Itoh M, Okuda S, Yoshizawa AC, Kanehisa M: KAAS: an automatic genome annotation and pathway reconstruction server. Nucl Acids Res 2007, 35:W182-W185.

62. Conesa A, Götz S: Blast2GO: a comprehensive suite for functional analysis in plant genomics. Int J Plant Genomics 2008, 2008:1-13.

63. Gambino G, Gribaudo I: Simultaneous detection of nine grapevine viruses by multiplex reverse transcription-polymerase chain reaction with coamplification of a plant RNA as internal control. Phytopathology 2006, 96:1223-1229.

64. Galetto L, Rashidi M, Yamchi A, Veratti F, Marzachì C: In Vitro Expression of Phytoplasma Immunodominant Membrane Proteins. In Phytoplasmas and Phytoplasma Diseases Management: how to Reduce Their Economic Impact. Edited by Bertaccini A. Bologna, Italy: IPWG - International Phytoplasmologist Working Group; 2014:272-279.

65. Osborn AM, Smith CJ: Molecular Microbial Ecology. New York, NY: Taylor \& Francis; 2005.

66. Gutha L, Casassa L, Harbertson J, Naidu R: Modulation of flavonoid biosynthetic pathway genes and anthocyanins due to virus infection in grapevine (Vitis vinifera L.) leaves. BMC Plant Biol 2010, 10:187.

doi:10.1186/1471-2164-15-1088

Cite this article as: Abbà et al.: RNA-Seq profile of flavescence dorée phytoplasma in grapevine. BMC Genomics 2014 15:1088. 